\title{
Hypertension Induced Psychosis: A Case Report
}

\section{Shiva Kumar Reddy Mukkamalla*, Insaf Ally, Khalid Mahmood and Vincent Rizzo}

Icahn School of Medicine at Mount Sinai/Queens Hospital Center, USA

Keywords: Hypertension; Hypertensive encephalopathy; Hypertensive emergency; Psychosis; Hypertensive psychosis

An estimated 68 million people in the United States suffer from hypertension, with more than 25,000 deaths per year attributable to uncontrolled hypertension [1]. Hypertension, although rare, is a wellknown cause of encephalopathy. In one of the previous studies it was found that encephalopathy constitutes about $16 \%$ of all end-organ complications which may arise from hypertensive crisis [2]. However, to the best of our knowledge, there have been no reported cases in the literature depicting hypertension as a direct cause of frank psychosis. Through this case report we will present a case of new onset psychosis in relation to hypertensive emergency and address the importance of considering hypertensive psychosis as a part of the differential in patients presenting with psychosis.

A 60 year-old African American male with a known history of sickle cell trait, hypertension, type 2 diabetes mellitus, coronary artery disease and chronic kidney disease stage IV was brought to the emergency center for chest pain of one day duration and strange behavior, as reported by family members, for three days. There was no report of any head injury or seizure activity prior to presentation to the emergency department.

Patient was recently discharged from the hospital after being treated for hyperglycemic hyperosmolar non-ketosis state and bacterial meningitis complicated by new onset seizures. At the time of discharge patient was clinically stable with no complaints of psychosis or delirium. However, one day prior to the aforementioned discharge, the patient had an episode of auditory and visual hallucinations which coincided with elevated blood pressure and resolved with optimum blood pressure control before being discharged home. Three days prior to the current admission, the patient started having formication along with visual and auditory hallucinations. He was seen to be chanting and praying, though he was never known to be a religious person. He was also writing prayers and Biblical sayings on the walls of his home, and speaking nonsensically. He was unable to sleep at home and was noted to be more aggressive, which was uncharacteristic of his behavior. The patient also reported seeing dead people moving around and feared that he was going to die. On the day of admission he was complaining of left sided chest tightness without any radiation to the arms or neck.

At the time of examination, the patient's blood pressure was 210/118 $\mathrm{mm} \mathrm{Hg}$. He was awake, alert and anxious. There was no papilledema nor were there any other signs of focal neurological deficit. Initial laboratory studies were positive for elevated troponins with no significant electrocardiographic changes. Serum glucose level was low ( $46 \mathrm{mg} / \mathrm{dL})$, which was corrected. There was no evidence of any acute hemorrhage, mass effect or edema noted on Computed Tomography (CT) scan of head. Patient was admitted with the following medical diagnoses: (1) hypertensive emergency, (2) rule out acute coronary syndrome, (3) altered mental status vs. psychosis, unspecified. Cardiology was consulted and patient was placed under close psychiatric observation.

Cardiology assessed the patient and the chest pain with elevated troponins was attributed to be secondary to hypertensive emergency and acute coronary syndrome was ruled out. Meanwhile, parenteral intravenous nitroglycerin was immediately administered along with clonidine per oral. The patient's blood pressure remained high at 183/92 mm Hg. He was transferred to medical floors with continued close psychiatric observation and was started on labetalol, clonidine, amlodipine, hydralazine, and isosorbide dinitrate. Gradually his blood pressure came down to $149 / 67 \mathrm{~mm} \mathrm{Hg}$ over the next 24 hours along with significant resolution of his psychosis. Magnetic Resonance Imaging (MRI) of brain was done, which failed to show any signs suggestive of edema or encephalopathy. However, MRI of brain did show tiny acute infarcts in central pons, which would not explain the patient's presentation.

A broad differential was laid down initially to explain this psychosis/altered mental status, which included hypoglycemia, hepatic encephalopathy, uremic encephalopathy, Central Nervous System (CNS) infection, CNS mass lesions and hypertensive encephalopathy. Hypoglycemia was ruled out since his psychosis did not improve with glucose levels followed by ruling out hepatic encephalopathy with normal liver function tests and ammonia levels. Pt. had chronic kidney disease with no interval change to support the idea of uremic encephalopathy. Among the tests that were done to rule out infectious etiologies the only significant one was that of West Nile Virus (WNV) IgG and IgM testing. However, it was noted that WNV IgG was positive while WNV IgM was negative, which depicted past flavivirus exposure with no active infection. CT head and MRI head were negative for CNS mass lesions. After excluding all other possible causes of psychosis/ altered mental status it was determined that patient has psychosis secondary to hypertensive emergency. Psychiatric evaluation was completed and it was opined that the psychosis was secondary to hypertensive emergency which did not require any antipsychotic medications.

Literature search was conducted in PUBMED using the MeSH terms "Hypertension" and "Psychotic Disorders". There were no studies done or cases reported relating hypertension to psychosis. To our knowledge this is the first case of hypertension related psychosis. However, the pathophysiology behind its development remains unclear. At this time we can only hypothesize the pathophysiology to be similar to that of hypertensive encephalopathy, i.e., loss of auto-regulatory mechanism of cerebral blood flow along with disruption of cerebrovascular endothelium [3].

Hypertensive psychosis might seemingly be very rare, but nevertheless physicians should understand and consider it to be

*Corresponding author: Shiva Kumar Reddy Mukkamalla, Icahn School of Medicine at Mount Sinai/Queens Hospital Center, USA, E-mail: shiva.mukkamalla@gmail.com

Received November 06, 2013; Accepted January 29, 2014; Published January 31,2014

Citation: Mukkamalla SKR, Ally I, Mahmood K, Rizzo V (2014) Hypertension Induced Psychosis: A Case Report. J Hypertens 3: 137. doi:10.4172/2167 1095.1000137

Copyright: () 2014 Mukkamalla SKR, et al. This is an open-access article distributed under the terms of the Creative Commons Attribution License, which permits unrestricted use, distribution, and reproduction in any medium, provided the original author and source are credited. 
a possible cause of new onset psychosis in a patient presenting with hypertensive crisis or emergency, once other common causes have been excluded. The role of antipsychotics in the treatment of hypertensive psychosis remains uncertain. Further investigation is warranted to determine the independent and/or synergistic role of antipsychotics along with anti-hypertensive medications in treatment of hypertension induced psychosis.

\section{References}

1. CDC, National Center for Health Statistics (2013) Hypertension.

2. Zampaglione B, Pascale C, Marchisio M, Cavallo-Perin P (1996) Hypertensive urgencies and emergencies. Prevalence and clinical presentation. Hypertension 27: $144-147$

3. Strandgaard S, Paulson OB (1989) Cerebral blood flow and its pathophysiology in hypertension. Am J Hypertens 2: 486-492. 